\title{
Levels of PEDF in pleural effusions from lung adenocarcinoma and benign disease patients
}

\author{
Nuria Sánchez-Otero ${ }^{\mathrm{a}}$, Sonia Blanco-Prieto ${ }^{\mathrm{a}}$, Lorena Vázquez-Iglesias ${ }^{\mathrm{a}}$, María Páez de la Cadena ${ }^{\mathrm{a}, *}$, \\ Alberto Fernández-Villar ${ }^{\mathrm{b}}$, María Isabel Botana-Rial ${ }^{\mathrm{b}}$ and Francisco Javier Rodríguez-Berrocal ${ }^{\mathrm{a}}$ \\ a Department of Biochemistry, Genetic and Immunology, Facultad de Biología, Universidad de Vigo, Vigo, Spain \\ ${ }^{\mathrm{b}}$ Unit of Interventional Bronchopleural Pathology, Pneumology Department of Complejo Hospitalario, \\ Universitario de Vigo, Vigo, Spain
}

\begin{abstract}
Anti-tumor properties assigned to PEDF, beside its role as an inhibitor of angiogenesis, make it a promising candidate in the search of new biomarkers for malignancy. In this study levels of PEDF were investigated in pleural effusions from lung adenocarcinoma and benign inflammatory disease patients. The mean PEDF concentration in the malignant group was slightly superior to that in patients suffering benign diseases $(4.59 \mu \mathrm{g} / \mathrm{mL}$ vs $3.97 \mu \mathrm{g} / \mathrm{mL})$, although the difference did not reach statistical significance $(P$ 0.166). Pleural effusion PEDF levels were not related to gender, age, smoking habit or pleural effusion size. We also investigated the possible relationship of PEDF levels in pleural effusion regarding clinicopathological features. Correlations were found for monocytes $(P$ 0.010) and polymorphonuclear leukocytes $(P 0.023)$ with PEDF levels in pleural effusion of malignant origin.
\end{abstract}

Keywords: PEDF, pleural effusion, biomarkers

\section{Introduction}

Pigment Epithelium Derived Factor (PEDF) is a $50 \mathrm{kDa}$ glycoprotein composed of 418 amino acids [1], that was first isolated from the conditioned medium of human fetal retinal pigment epithelial cells as a inducer of neuronal differentiation and maturation of retinoblastoma cells [2,3]. PEDF belongs to the serine protease inhibitor (serpin) family of proteins but lacks homology at the serpin reactive center, demonstrating no antiprotease activity [1]. The PEDF gene is highly conserved among mammalian species [4] and its expression has been proved in a wide range of fetal and adult tissues, including the spinal cord, cerebrospinal fluid, skeletal muscle [5], bone [6], heart, liver, testis, ovaries, placenta, pancreas and lung [4] and in plasma [7].

\footnotetext{
*Corresponding author: María Páez de la Cadena, Department of Biochemistry, Genetic and Immunology, Facultad de Biología, Universidad de Vigo, 36310 Vigo, Spain. E-mail: mpaez@uvigo.es.
}

PEDF exhibits multiple and varied biological activities, it is a potent antiangiogenic factor inhibiting endothelial cell migration and inducing apoptosis of activated endothelial cells [8,9], and neuroprotective properties have too been reported [10].

In tumors, in addition to its antiangiogenic activity, PEDF has a role as a tumor-cell differentiator and inducer of tumor-cell apoptosis [11]. Reduced expression of PEDF has been demonstrated in several tumors correlated with a poor prognosis, a more metastatic phenotype and higher microvessel density, including neuroblastoma [10], neoplasias of the prostate [12, 13], lung [14,15], breast [16], bladder [17] and pancreas [18]. Since angiogenesis is essential for tumors to grow and metastasize, the antiangiogenic activity of PEDF makes it a promising target for cancer therapy [19]. Effectiveness of intrapleural gene transfer of PEDF resulting in inhibition of tumor growth and prolonged survival has been reported in a murine model of lung cancer [20], for instance.

Pleural effusion (PE), an accumulation of fluid in the pleural cavity surrounding the lungs, results 
from an increased production exceeding fluid removal rate [21]. Pleural effusions are classified as transudates or exudates according to the biochemical characteristics of the fluid, but their distinction is easily achieved by the use of Light's criteria [22]. A common cause of exudative PE is the presence of any malignancy, and so far the challenge is the discrimination between malignant PE (MPE) and benign PE (BPE) [22, 23]. Unfortunately, methodologies used in the diagnosis of malignant PE (MPE) lack proper sensitivity, the case for cytology analysis with sensitivities ranging from 40 to $87 \%$ [24], or are highly invasive like thoracoscopy [25]. In this setting, the use of tumor markers constitutes the non-invasive alternative to complement these techniques; however, to date assayed markers have not proved sensitive and specific enough to diagnose MPE patients [26,27].

In a previous proteomic study, comparing the PE proteome profiles of NSCLC versus tuberculosis patients, 35 proteins were identified with significantly different levels between MPE and BPE. Among those proteins, two distinct isoforms of PEDF were overexpressed in PE from cancer patients [28]. This finding, in conjunction with the anti-tumoral role attributed to PEDF [11], prompted us to explore the potential clinical significance of PEDF expression in MPE. In the current study, we have determined PEDF levels in pleural fluid of MPE derived from non-small cell lung cancer (NSCLC) and benign PE (BPE) derived from tuberculosis and parapneumonic affectations, exploring its possible diagnostic utility in MPE and the correlation of this biomarker with clinical and biochemical variables.

\section{Methods}

\subsection{Study population}

A total of 52 consecutive non-selected patients with a specific diagnosis for exudative PE were enrolled in this prospective study. Patients were admitted from April 2007 to December 2010 in the Unit of Interventional Bronchopleural Pathology, at the Pneumology Department of Complejo Hospitalario Universitario de Vigo (CHUVI).

The study of PE samples followed the clinicalethical practices of the Spanish Government; the protocol of this study was approved by the Galician Ethical Committee for Clinical Research (2007/179), and complied with the Helsinki Declaration, Oviedo
Agreement, the Organic Law for Data Protection 15/1999, and Royal Decree 1720/2007. All participants gave written informed consent to provide PE samples and using clinical history numbers anonymity was warranted.

\subsection{Pleural fluid diagnosis}

Diagnosis of patients was established performing a thoracocentesis that allows for the biochemical, microbiological and cytological analyses of the pleural fluid. Achievement of a diagnosis without the need of performing a thoracocentesis was considered as an exclusion criterion, establishing inclusion criteria an accurate diagnosis of $\mathrm{PE}$ and the exudative nature of the effusion. When a diagnosis was not obtained after the first test, a second thoracocentesis and a blind percutaneous pleural biopsy with Abrams needle were conducted [23].

The biochemical parameters of pleural fluid were analyzed before the beginning of any treatment [differential cell counts, $\mathrm{pH}$, proteins, lactate dehydrogenase (LDH), glucose, and adenosinedeaminase (ADA)]. In the case several thoracocentesis had been performed, only the biochemical results of the first procedure were evaluated. The demographic variables of patients and the size of PE were too registered; PE extension was classified depending on the affectation of the hemithorax on the chest radiograph.

For the analysis we established two principal groups (MPE and BPE). PE was considered malignant when we could demonstrate malignant cells at cytological examination. Patients with lung adenocarcinoma formed this group. Patients with BPE were divided into three subgroups: tuberculous, pneumonic and idiopathic PE. Tuberculosis diagnosis was based on the presence of positive stain or culture for Mycobacterium tuberculosis in the pleural fluid, sputum, or pleural biopsy; and on the presence of typical caseating granulomas in pleural biopsy. Patients diagnosed with pneumonic effusion presented pneumonia symptoms and response to antibiotics. A last group of non-neoplasic PE of unknown origin was included as idiopathic, comprising non-specific pleuritis observed at thoracoscopy, thoracotomy, biopsy, or autopsy.

\subsection{Pleural effusion sampling}

Approximately $10 \mathrm{~mL}$ of pleural fluid of each patient were obtained at the same time thoracocentesis or pleural biopsy was done. Samples were centrifuged at $800 \mathrm{~g}$ for 15 minutes, and immediately frozen in $0.5 \mathrm{~mL}$ aliquots at $-20^{\circ} \mathrm{C}$ until their analysis. 
Table 1

Demographic data and values of PEDF in pleural fluid

\begin{tabular}{lccc}
\hline PE origin & No. of patients & PEDF, $\mu \mathrm{g} / \mathrm{mL}$ Mean (range) & $P$ Value \\
\hline MPE & 22 & & \\
Lung adenocarcinoma & & $4.59(1.95-7.37)$ & 0.166 \\
BPE & 27 & $3.97(1.18-6.43)$ \\
Tuberculous & 2 & $4.15(1.18-6.43)$ \\
Parapneumonic & 1 & $2.34(2.16-2.52)$ \\
Idiopathic & 2.62 & \\
\hline
\end{tabular}

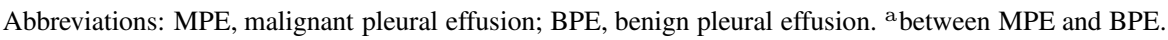

\subsection{Biochemical procedures}

We determined the levels of PEDF using a sandwich enzyme-linked immunosorbent assay (ELISA) commercially available from Chemicon International (USA and Canada). Laboratory PEDF test performance was completely independent of clinical diagnosis and treatment. The assay was conducted according to manufacturer's instructions. Since PEDF can be complexed with other proteins that may interfere in the quantification, pleural fluid samples were pretreated with urea to a final concentration of $8 \mathrm{M}$ and incubated in ice for 1 hour to release PEDF from its bound proteins. Urea-treated samples were diluted 1:500 in assay diluent and $100 \mu \mathrm{L}$ immediately applied to the plate (to minimize reassociation of the antigen to binding proteins). One hundred $\mu \mathrm{L}$ of each Standard were added to each well and the plate was incubated for 1 hour at $37^{\circ} \mathrm{C}$. Standards and samples were run in duplicate. The plate was washed four times with $250 \mu \mathrm{L}$ of wash buffer, $100 \mu \mathrm{L}$ of biotinylated Mouse Anti-Human PEDF monoclonal antibody were added and incubated for 1 hour at $37^{\circ} \mathrm{C}$. The washing procedure was repeated and $100 \mu \mathrm{L}$ of streptavidin-peroxidase conjugate were added to each well and incubate at $37^{\circ} \mathrm{C}$ for 1 hour again. A last washing procedure was performed and $100 \mu \mathrm{L}$ of tetramethylbenzidine enzyme (TMB/E) substrate were added, allowing the signal to develop for 5-10 minutes in dark at room temperature. The reaction was stopped by addition of $100 \mu \mathrm{L}$ of stop solution. Optical density was read at $450 \mathrm{~nm}$ on an EnVision Multilabel Plate Reader (Perkin Elmer).

The minimal detectable concentration of this assay was $0.9 \mathrm{ng} / \mathrm{mL}$, as indicated by the manufacturer.

\subsection{Statistical analysis}

Kolmogorov-Smirnov and Levene's tests were applied to continuous variables to verify normal distribution and homogeneity of variances, respectively. The Student t-test was used to evaluate differences between independent samples in normally distributed variables, while the Mann Whitney U test was used instead when normality was not confirmed. The bivariate relationships between the biochemical parameters and PEDF levels were assessed by Tau of Kendall correlation analysis.

PEDF ability to discriminate between MPE and BPE was evaluated by receiver operating characteristic (ROC) curves. Sensitivity, specificity, likelihood ratios (LRs) and their confidence intervals (CI), positive predictive value (PPV), negative predictive value (NPV) and diagnostic accuracy were calculated. To estimate positive LR the formula sensitivity/(1-specificity) was applied and the negative LR was calculated as (1sensitivity)/specificity. The cut-off point was chosen as the value that rendered the highest accuracy value.

All statistical analyses were performed with SPSS version 15.0 (SPSS Inc, Chicago, Illinois). $P$ values $<$ 0.05 were considered statistically significant. Sensitivity, specificity and LR were calculated using MedCalc version 11.5.1 (MedCalc Software, Belgium).

\section{Results and discussion}

A total of 52 patients diagnosed with PE were included in this study. According to the diagnostic criteria described in Methods, patients were grouped for statistical analysis as summarized in Table 1. In this cohort, MPE group corresponded to 22 patients presenting lung adenocarcinoma, while the BPE sample comprised 30 patients, most of them suffering tuberculosis (27 patients), the remainder patients presenting parapneumonic PE (2) and idiopathic PE (1).

The concentration of PEDF $(\mu \mathrm{g} / \mathrm{mL})$ in the different diagnostic groups is displayed in Table 1. The mean PEDF level in the malignant group was $4.59 \mu \mathrm{g} / \mathrm{mL}$, slightly superior to that in BPE patients, $3.97 \mu \mathrm{g} / \mathrm{mL}$, although the difference did not reach statistical significance $(P<0.166)$.

To date studies measuring PEDF levels in pleural fluid are very scarce. Although some researchers have 
Table 2

Cut-off, sensitivity, specificity, predictive values, LR+, LR - and accuracy for PEDF in classifying different PE groups

\begin{tabular}{lcccccc}
\hline $\begin{array}{l}\text { Group } \\
\text { and cut-off }\end{array}$ & $\begin{array}{c}\text { Sensitivity \% } \\
(95 \% \mathrm{CI})\end{array}$ & $\begin{array}{c}\text { Specificity \% } \\
(95 \% \mathrm{CI})\end{array}$ & PPV \% & NPV \% & $\begin{array}{c}\text { Positive LR } \\
(95 \% \mathrm{CI})\end{array}$ & $\begin{array}{c}\text { Negative LR } \\
(95 \% \mathrm{CI})\end{array}$ \\
\hline MPE vs. BPE & 59.09 & 63.33 & 54.17 & 67.86 & 1.61 & 0.65 \\
$\leqslant 4.25 \mu \mathrm{g} / \mathrm{mL}$ & $(36.4-79.3)$ & $(43.9-80.1)$ & & & 61.54 \\
$(1.0-2.5)$ & $(0.3-1.3)$ \\
\hline
\end{tabular}

$\mathrm{MPE}=$ malignant pleural effusion, $\mathrm{BPE}=$ benign pleural effusion, $\mathrm{CI}=$ confidence interval, $\mathrm{PPV}=$ positive predictive value, $\mathrm{NPV}=$ negative predictive value, $\mathrm{LR}=$ likelihood ratio.

applied proteomic technologies identifying PEDF as a component of the protein content of pleural effusion [29], few have focused on the comparison between malignant and benign exudates.

In the proteomic study of Rodríguez Piñeiro et al. [28] multiple isoforms of PEDF in both malignant and benign samples analysed were detected. They found two PEDF spots with increased ratios of 1.5 and 2 in MPE against tuberculous PE, using a small sample size. They performed an initial validation step by means of a non-quantitative strategy, describing multiple isoforms of PEDF but maintaining an overall higher expression in MPE. In the present work, levels of PEDF were determined using a specific ELISA method detecting total levels of the protein. Quantitative results in a larger population presented here corroborate the higher levels of PEDF in lung cancer malignancy, although significant differences are not longer maintained.

Using a similar proteomic approach to [28], Wang et al. [30], reported a 4.45 increase in PEDF levels in PE of lung adenocarcinoma origin when compared against pneumonic PE. Conversely, differences were not maintained when the benign PE was formed of tuberculosis effusions. These data are in agreement with the results presented here, where tuberculosis PE exhibits closer PEDF levels to MPE than pneumonic PE. Unfortunately, these authors did not perform a further validation of PEDF levels in PE. To our knowledge, only Hsieh et al. [31] determined PEDF concentration in malignant exudative effusions of diverse cancer origin, but comparing them to transudative effusions instead of benign exudative effusions. Although they found differences in PEDF levels, their results are not comparable to this study because of the different nature of samples processed.

Having no reference of the magnitude of PEDF levels in PE, we sought whether PEDF protein concentration reported in our study was maintained in serum or plasma of other studies. Noticeably, the PEDF concentration found by us in pleural fluid matches the range of levels described in plasma by other authors, $5 \mu \mathrm{g} / \mathrm{mL}$ on average [7,32]. Much of the interest of PE as a
Table 3

Pleural fluid values of PEDF according to the clinic pathological features

\begin{tabular}{lccc}
\hline Characteristics & $\begin{array}{c}\text { No. of } \\
\text { patients }\end{array}$ & $\begin{array}{c}\text { PEDF mean } \\
(\text { range })(\mu \mathrm{g} / \mathrm{ml})\end{array}$ & $P$ value \\
\hline Gender & 33 & $4.15(1.18-7.04)$ & 0.602 \\
$\quad$ Male & 19 & $4.38(1.61-7.36)$ & \\
$\quad$ Female & & & \\
Age & 25 & $3.92(1.61-6.43)$ & 0.164 \\
$\quad \leqslant 50$ & 27 & $4.53(1.18-7.36)$ & \\
$\quad>50$ & & & \\
Smoking habits & 21 & $4.36(1.61-7.01)$ & 0.435 \\
$\quad$ Non-smoker & 29 & $4.02(1.18-7.04)$ & \\
$\quad$ Smoker and ex-smoker & & & \\
$\quad$ PE Size & 13 & $4.07(1.95-6.43)$ & 0.661 \\
$\quad<1 / 3$ & 39 & $4.29(1.18-7.36)$ & \\
$\quad>1 / 3$ & & & \\
\hline
\end{tabular}

source of biomarkers for malignancy derives from its proximity to the affected organ and the smaller dilution effect in comparison to other body fluids; therefore, serum and plasma do not always reflect what happens in other tissues. In this case, we considered interesting that PEDF levels were maintained in the pleural fluid.

As expected from the results described in Table 1, the diagnostic performance of PEDF levels proved not useful in discriminating malignant PE origin (Table 2). The area under the ROC curve (AUC) was $0.6(95 \%$ CI: $0.455-0.733)$. A cut-off value of $4.25 \mu \mathrm{g} / \mathrm{mL}$ was established as it provided the highest accuracy, providing sensitivity and specificity parameters of 59.09 and $63.33 \%$, respectively; predictive values, likelihood ratios and accuracy are also displayed on Table 2. Nevertheless, some discriminating value has been assigned to PEDF in other malignancies; Byrne et al. [13] reported a better but still not acceptable discrimination value in prostate cancer from benign hyperplasia with an AUC of 0.788.

The absence of striking differences between malignant and benign PE analysed in this work may reside on the nature of the samples compared and the multiplicity of PEDF functions. On the one hand, higher PEDF levels in cancerous PE could be expected as an attempt to limit tumoral damage, as seen in amyotrophic lateral sclerosis [33]. On the other 
hand, benign samples represent inflammatory conditions and PEDF has also been reported to have antiinflammatory properties [34].

The possible relationship of PEDF levels in PE regarding clinicopathological features of the patients was also investigated (Table 3). No differences in PEDF levels were encountered in relation to gender, age, smoking habit or PE size. Referring age and gender, results agree with previous works from Feng et al. [17] and Uehara et al. [18], in bladder tumor and pancreatic carcinoma respectively. We also assessed the correlation between PEDF levels and other variables. No significant correlation was found with levels of adenosine desaminase, lactate dehydrogenase, protein content, glucose, and $\mathrm{pH}$ values (data not shown). Nevertheless, significative correlations were found for monocytes $(P 0.010)$ and polymorphonuclear leukocytes $(\mathrm{PMN})(P$ 0.023) with PEDF levels in MPE samples, applying Tau of Kendall test.

The positive correlation of PEDF with PMN and monocytes in MPE samples is in agreement with the finding of Bard et al. [35]. These authors reported for the first time PEDF as a component of exosomes, membrane vesicles secreted both by lymphocytic and tumoural cells, isolated in cancerous pleural fluid. Although it is known that exosomes elicit anti-tumoral responses, the role of PEDF in exosomes is not clear.

\section{Conclusion}

We have for the first time reported quantitative expression levels of PEDF comparing PE from malignant and benign origin, by the application of specific immunoquantification methodologies of protein, demonstrating in both samples high levels of PEDF coincident with the concentration described in serum. Disappointingly, the levels of this protein do not allow an accurate discrimination of $\mathrm{PE}$ origin. Involvement of PEDF in several functionalities, comprising both tumorigenesis and inflammation, could abrogate its diagnostic accuracy when pretending to discern cancerous from benign samples, mostly inflammatory benign samples.

\section{Acknowledgments}

This research was partially supported by grants PS09-00405 and Research Intensification activity from Fondo de Investigación Sanitaria (FIS) of the Instituto de Salud Carlos III (Spain), and Xunta de Galicia and FEDER founding (CN 2011/024). Sonia Blanco is supported by a fellowship from the Programa Nacional de Formación de Profesorado Universitario (FPU, Ministerio de Ciencia e Innovación of Spain). Special thanks to L. Barcia for their assistance. We are grateful to V. Leiro, C. Represas and M. Núñez (Pneumology Department, CHUVI)) for their collaboration in the acquisition of samples.

\section{References}

[1] F.R. Steele, G.J. Chader, L.V. Johnson and J. Tombran-Tink. Pigment epithelium-derived factor: neurotrophic activity and identification as a member of the serine protease inhibitor gene family. Proc Natl Acad Sci U S A 90 (1993), 1526-1530.

[2] J. Tombran-Tink and L.V. Johnson. Neuronal differentiation of retinoblastoma cells induced by medium conditioned by human RPE cells. Invest Ophthalmol Vis Sci 30 (1989), 17001707.

[3] J. Tombran-Tink, G.G. Chader and L.V. Johnson. PEDF: a pigment epithelium-derived factor with potent neuronal differentiative activity. Exp Eye Res 53 (1991), 411-414.

[4] J. Tombran-Tink, K. Mazuruk, I.R. Rodriguez, D. Chung, T. Linker, E. Englander and G.J. Chader. Organization, evolutionary conservation, expression and unusual Alu density of the human gene for pigment epithelium-derived factor, a unique neurotrophic serpin. Mol Vis 4 (1996), 2-11.

[5] M.M. Bilak, A.M. Corse, S.R. Bilak, M. Lehar, J. TombranTink and R.W. Kuncl. Pigment epithelium-derived factor (PEDF) protects motor neurons from chronic glutamatemediated neurodegeneration. J Neuropathol Exp Neurol 58 (1999), 719-28.

[6] G.M. Quan, J. Ojaimi, Y. Li, V. Kartsogiannis, H. Zhou and P.F. Choong. Localization of pigment epithelium-derived factor in growing mouse bone. Calcif Tissue Int 76 (2005), 146153.

[7] S.V. Petersen, Z. Valnickova and J.J. Enghild. Pigmentepithelium-derived factor (PEDF) occurs at a physiologically relevant concentration in human blood: purification and characterization. Biochem J 374 (2003), 199-206.

[8] D.W. Dawson, O.V. Volpert, P. Gillis, S.E. Crawford, H. Xu, W. Benedict and N.P. Bouck.Pigment epithelium derived factor: a potent inhibitor of angiogenesis. Science 285 (1999), 245-248.

[9] V. Stellmach, S.E. Crawford, W. Zhou and N. Bouck. Prevention of ischemia-induced retinopathy by the natural ocular antiangiogenic agent pigment epithelium-derived factor. PNAS 98 (2001), 2593-2597.

[10] S.E. Crawford, V. Stellmach, M. Ranalli, X. Huang, L. Huang, O. Volpert, G.H. De Vries, L.P. Abramson and N. Bouck. Pigment epithelium-derived factor (PEDF) in neuroblastoma: a multifunctional mediator of Schwann cell antitumor activity. J Cell Sci 114 (2001), 4421-4428.

[11] E.T.H. EK, C.R. Dass and P.F.M. Chong. Pigment epitheliumderived factor: a multimodal tumor inhibitor. Mol Cancer Ther 5 (2006), 1641-1646.

[12] S. Halin, P. Wikstrom, S.H. Rudolfsson, P. Stattin, J.A. Doll, S.E. Crawford and A. Bergh. Decreased pigment epitheliumderived factor is associated with metastatic phenotype in hu- 
man and rat prostate tumors. Cancer Res 64 (2004), 56645671 .

[13] J.C. Byrne, M.R. Downes, N. O'Donoghue, C. O'Keane, A O’Neill, Y. Fan, J.M. Fitzpatrick, M. Dunn and R.W. Watson. 2D-DIGE as a Strategy to identify serum markers for the progression of prostate cancer. J Proteome Res 8 (2009), 942957.

[14] L. Zhang, J. Chen, Y. Ke, R.E. Mansel and W.G. Jiang. Expression of pigment epithelial derived factor is reduced in non-small cell lung cancer and is linked to clinical outcome. Int J Mol Med 17 (2006), 937-944.

[15] J. Chen, L. Ye, L. Zhang and W.G. Jiang. The molecular impact of pigment epithelium-derived factor, PEDF, on lung cancer cells and the clinical significance. Int J Oncol 35 (2009), 159-166.

[16] D. Zhou, S.Q. Cheng, H.F. Ji, J.S. Wang, H.T. Xu, G.Q. Zhang and D. Pang. Evaluation of protein pigment epitheliumderived factor (PEDF) and microvessel density (MVD) as prognostic indicators in breast cancer. J Cancer Res Clin Oncol 136 (2010), 1719-1727.

[17] C.C. Feng, Q. Ding, Y.F. Zhang, H.W. Jiang, H. Wen, P.H. Wang and $\mathrm{Z}$. Wu. Pigment epithelium-derived factor expression is down-regulated in bladder tumors and correlates with vascular endothelial growth factor and matrix metalloproteinase-9. Int Urol Nephrol 43 (2011), 383-390.

[18] H. Uehara, M. Miyamoto, K. Kato, Y. Ebihara, H. Kaneko, H. Hashimoto, Y. Murakami, R. Hase, R. Takahashi, S. Mega, T. Shichinohe, Y. Kawarada, T. Itoh, S. Okushiba, S. Kondo and $\mathrm{H}$. Katoh. Expression of pigment epithelium-derived factor decreases liver metastasis and correlates with favorable prognosis for patients with ductal pancreatic adenocarcinoma. Cancer Res 64 (2004), 3533-3537.

[19] K.B. Manalo, P.F. Choong and C.R. Dass. Pigment epithelium-derived factor as an impending therapeutic agent against vascular epithelial growth factor-driven tumorangiogenesis. Mol Carcinog 50 (2011), 67-72.

[20] A. Mahtabifard, R.E. Merritt, R.E. Yamada, R.G. Crystal and R.J. Korst. In vivo gene transfer of pigment epitheliumderived factor inhibits tumor growth in syngeneic murine models of thoracic malignancies. J Thorac Cardiovasc Surg 126 (2003), 28-38.

[21] G. Miserocchi. Physiology and pathophysiology of pleural fluid turnover. Eur Respir J 10 (1997), 219-225.

[22] Heffner, JE. Diagnosis and management of malignant pleural effusion. Respirology 13 (2008), 5-20.

[23] V. Villena Garrido, J. Ferrer Sancho, L. Hernández Blasco, A. de Pablo Gafas, E. Pérez Rodríguez, F. Rodríguez Panadero, S. Romero Candeira, A. Salvatierra Velázquez and L. Valdés Cuadrado L. Diagnosis and treatment of pleural effusion. Arch Bronconeumol 42 (2006), 349-372.

[24] N.A. Maskell and R.J. Butland. BTS guidelines for the investigation of a unilateral pleural efusion in adults. Thorax $\mathbf{5 8}$ (2003), ii8-ii17.
[25] R.W. Light. The undiagnosed pleural effusion. Clin Chest Med 27 (2006), 309-319.

[26] J.M. Porcel, M. Vives, A. Esquerda, A. Salud, B. Pérez and F. Rodríguez-Panadero. Use a panel of tumor markers (carcinoembryonic antigen, cancer antigen 125 , carbohydrate antigen 15-3, and cytokeratin 19 fragments) in pleural fluid for the differential diagnosis of benign and malignant Effusions. Chest 126 (2004), 1757-1763.

[27] F. Ferrer, M.A. Villarino, G. Encabo, E. Felip, B. Bermejo, S. Vilà and R. Orriols. Diagnostic utility of CYFRA 21-1, carcinoembryonic antigen, CA 125, neuron specific enolase, and squamous cell antigen level determinations in the serum and pleural fluid of patients with pleural effusions. Cancer $\mathbf{8 6}$ (1999), 1488-1495.

[28] A.M. Rodríguez-Piñeiro, S. Blanco-Prieto, N. Sánchez-Otero, F.J. Rodríguez-Berrocal and M. Páez de la Cadena. On the identification of biomarkers for non-small cell lung cancer in serum and pleural effusion. J Proteomics 73 (2010), 15111522.

[29] Y.C. Tyan, H.Y. Wu, W.W. Lai, W.C. Su and P.C. Liao. Proteomic profiling of human pleural effusion using twodimensional nano liquid chromatography tandem mass spectrometry. J Proteome Res 4 (2005), 1274-86.

[30] Z. Wang, C. Wang, X. Huang, Y. Shen, J. Shen and K. Ying. Differential proteome profiling of pleural effusions from lung cancer and benign inflammatory disease patients. Biochim Biophys Acta. 2012 Apr; 1824(4) (2012 Feb 2), 692-700.

[31] W.Y. Hsieh, M.W. Chen, H.T. Ho, T.M. You and Y.T. Lu. Identification of differentially expressed proteins in human malignant pleural effusions. Eur Respir J 28 (2006), 1178-1185.

[32] N. Ogata, M. Matsuoka, K. Matsuyama, C. Shima, A. Tajika, T. Nishiyama, M. Wada, N. Jo, A. Higuchi, K. Minamino, H. Matsunaga, T. Takeda and M. Matsumura. Plasma concentration of pigment epithelium-derived factor in patients with diabetic retinopathy. J Clin Endocrinol Metab 92 (2007), 11761179.

[33] T. Falk, R.T. Gonzalez, S.J. Sherman. The Yin and Yang of VEGF and PEDF: Multifaceted neurotrophic factors and their potential in the treatment of parkinson's disease. Int J Mol Sci 11 (2010), 2875-2900.

[34] S. Yamagishi, H. Adachi, A. Abe, T. Yashiro, M. Enomoto, K. Furuki, A. Hino, Y. Jinnouchi, K. Takenaka, T. Matsui, K. Nakamura and T. Imaizumi. Elevated serum levels of pigment epithelium-derived factor (PEDF) in the metabolic syndrome. J Clin Endocrin Metab 91 (2006), 2447-2450.

[35] M.P. Bard, J.P. Hegmans, A. Hemmes, T.M. Luider, R. Willemsen, L.A. Severijnen, J.P. van Meerbeeck, S.A. Burgers, H.C. Hoogsteden and B.N. Lambrecht. Proteomic Analysis of Exosomes Isolated from Human Malignant Pleural Effusions. Am J Respir Cell Mol Biol 31 (2004), 114-121. 


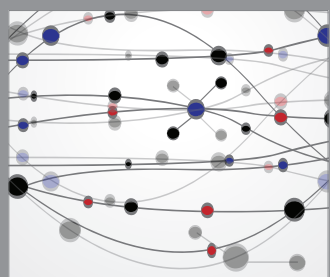

The Scientific World Journal
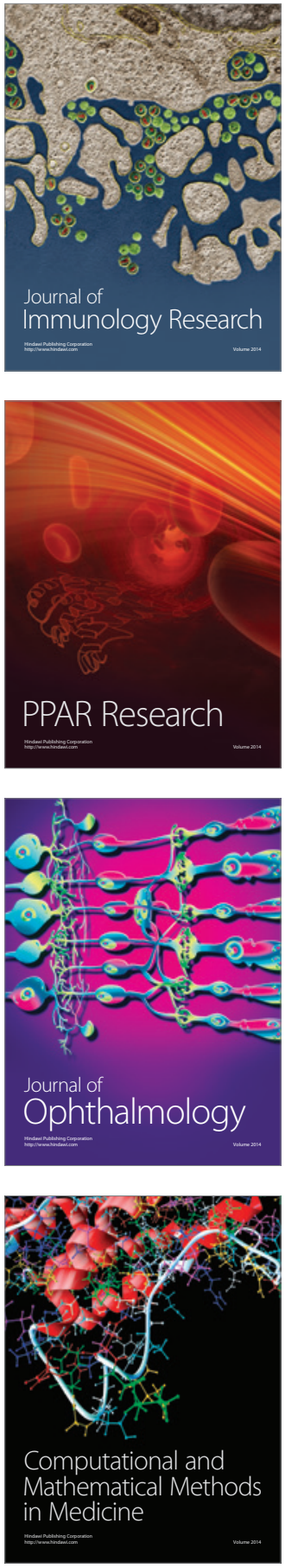

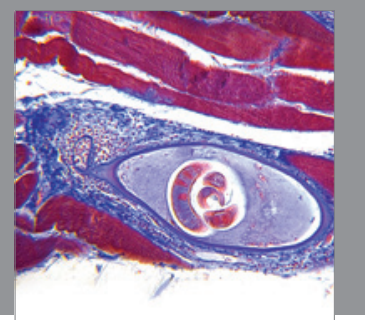

Gastroenterology

Research and Practice
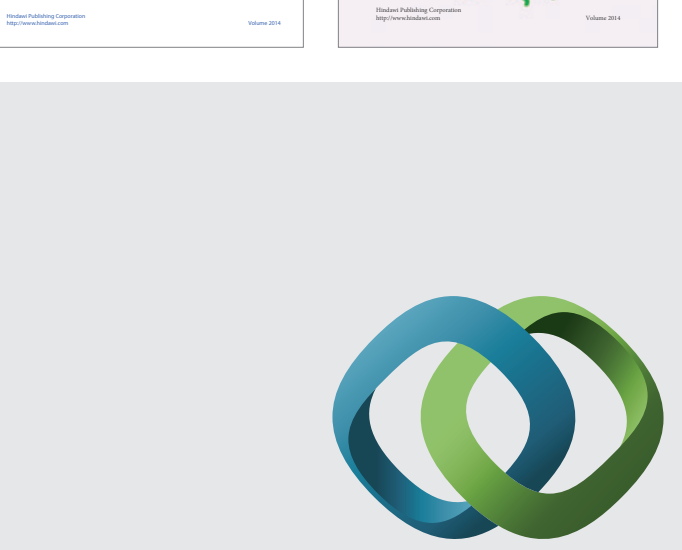

\section{Hindawi}

Submit your manuscripts at

http://www.hindawi.com
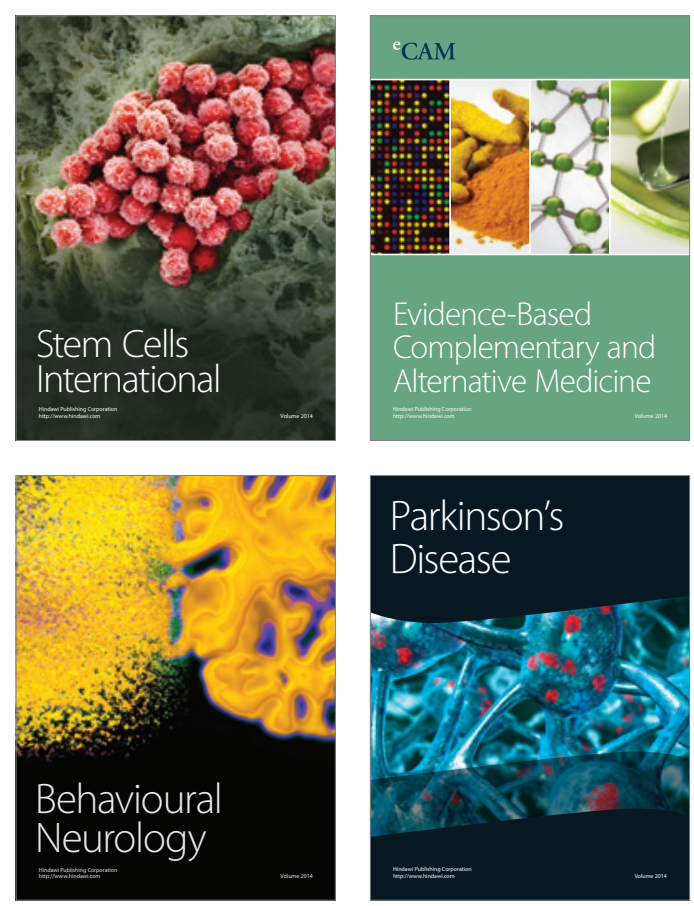

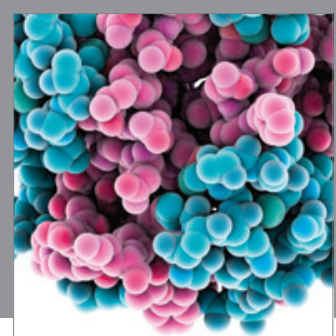

Journal of
Diabetes Research

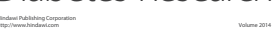

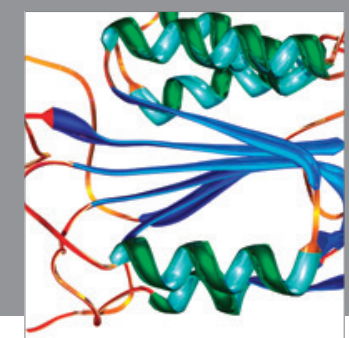

Disease Markers
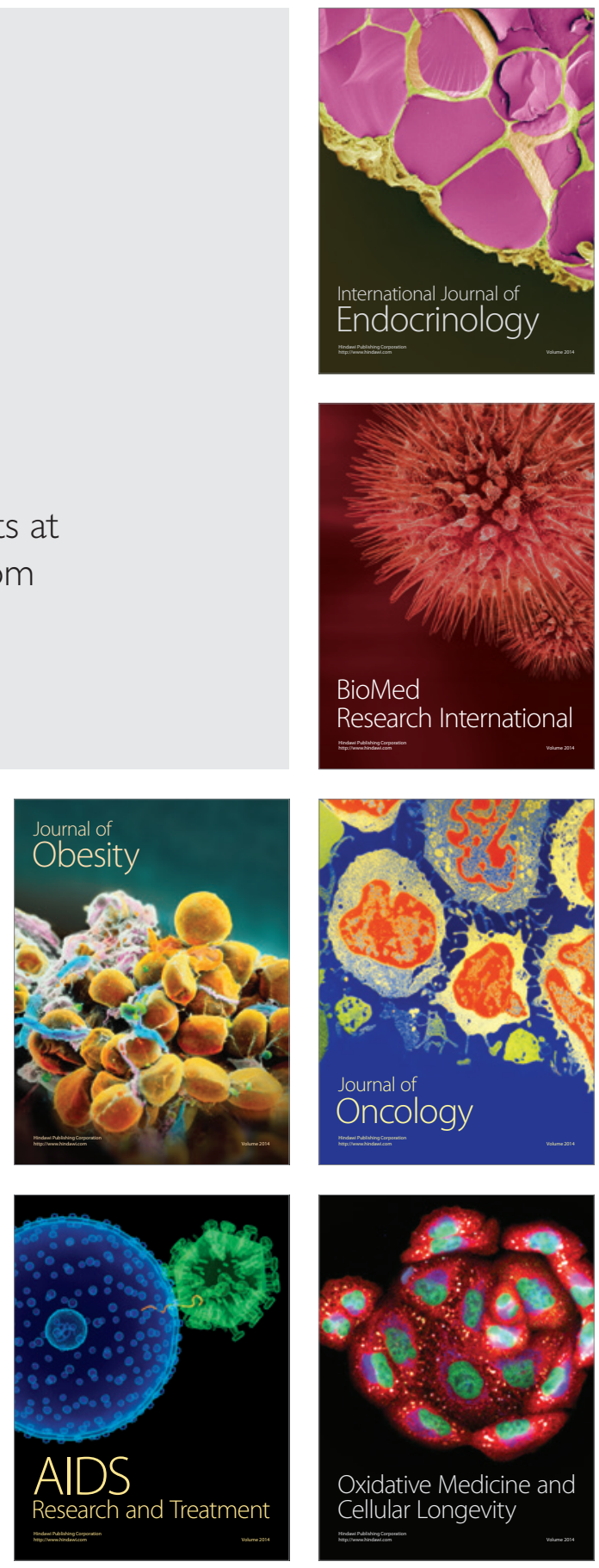